\title{
Fatal anaphylaxis to intravenous co-amoxiclav: A case series
}

\author{
Niall P Conlon, J David M Edgar
}

\begin{abstract}
Introduction: Fatal anaphylaxis to co-amoxiclav is rarely reported. We report three fatalities directly attributable to intravenous coamoxiclav administration. Case Report: Three patients with no prior history of drug allergy developed fatal anaphylaxis following intravenous administration of co-amoxiclav. Notably two of the three cases had been administered co-amoxiclav in the weeks preceding the fatal event. Each patient also had a factor associated with increased severity of anaphylaxis. Despite timely, appropriate and aggressive management of the anaphylactic reactions each case had a fatal outcome. Conclusion: Fatal anaphylaxis to co-amoxiclav is uncommon. These cases are typical of the rapid symptom onset of type I hypersensitivity to parenteral medication. Each incident occurred within a four-year time frame in a relatively small geographic area. This is out of proportion to 10 other fatalities related to amoxicillin containing medications occurring in the UK
\end{abstract}

Niall P Conlon ${ }^{1}$, J David M Edgar²

Affiliations: ${ }^{1} \mathrm{MB}$ BCh, MRCP(UK), Regional Immunology Service, Belfast Health and Social Care Trust, Elliott Dynes Unit, Royal Victoria Hospital, 273 Grosvenor Road, Belfast, UK, BT12 6BA; ${ }^{2} \mathrm{MB}$ BCh, FRCP(UK), FRCPath, Regional Immunology Service, Belfast Health and Social Care Trust, Elliott Dynes Unit, Royal Victoria Hospital, 273 Grosvenor Road, Belfast, UK, BT12 6BA.

Corresponding Author: Niall $\mathrm{P}$ Conlon, $\mathrm{MB} \mathrm{BCh}, \mathrm{BSc}$. (Hons.) MRCP (UK) Regional Immunology Service, Belfast Health and Social Care Trust, Elliott Dynes Unit, Royal Victoria Hospital, 273 Grosvenor Road, Belfast, UK, BT12 6BA; Ph: 00442890630003; Fax: 00442890635482; Email: conlonn1@tcd.ie

Received: 19 July 2012

Accepted: 17 October 2012

Published: 01 April 2013 over a 47-year period. The clinical diagnoses were supported by elevations in serum mast cell tryptase. Assessment of drug allergy commonly relies on clinical history supported by skin and intradermal testing. Risk assessment algorithms incorporating such testing techniques are unlikely to have altered outcome in these cases. These cases indicate that anaphylaxis to co-amoxiclav may have a catastrophic outcome despite immediate management. Such cases may be under reported and more prevalent than previously indicated. All staff administering parenteral co-amoxiclav should be aware of the risk of anaphylaxis even in patients with a prior history of tolerance of the drug.

Keywords: Anaphylaxis, Drug allergy, Coamoxiclav, Mast cell tryptase

$$
* * * * * * * * *
$$

Conlon NP, Edgar JDM. Fatal anaphylaxis to intravenous co-amoxiclav: A case series. International Journal of Case Reports and Images 2013;4(4):190-193.

$$
* * * * * * * * *
$$

doi:10.5348/ijcri-2013-04-292-CS-1

\section{INTRODUCTION}

Anaphylaxis is defined as a serious allergic reaction that is rapid in onset and has the potential to cause fatality [1]. In the health care setting, medication related anaphylaxis is an important complication of pharmacological therapy. Antibiotics, including $\beta$-lactams, are an important cause of in-hospital anaphylaxis [2]. Deaths from such reactions are considered rare [3]. However, fatal anaphylaxis may be under reported due to underdiagnosis by health care 
professionals, insensitive and non specific laboratory tests and the absence of clear postmortem findings [4]. We report three cases of fatal anaphylaxis related to the administration of intravenous (IV) co-amoxiclav. These cases highlight the unpredictable nature of anaphylaxis related to parenteral co-amoxiclav administration.

\section{CASE REPORT}

Case 1: A 52-year-old female, with a background of rheumatoid arthritis and mild asthma, but no history of drug allergy, was admitted to hospital with severe lower abdominal pain. She had been discharged from hospital 13 days before with following treatment with IV metronidazole and IV co-amoxiclav for a sigmoid diverticular abscess. On second admission a computed tomography (CT) scan showed an inflammatory diverticular mass in the right iliac fossa. Treatment with cefotaxime and metronidazole was commenced with a good therapeutic response. On day 5 of IV antibiotic therapy the patient was erroneously administered $1.2 \mathrm{~g}$ co-amoxiclav intravenously. During slow IV administration of the co-amoxiclav the patient complained of a strange taste in her mouth. Administration was discontinued before completion of the dose. However, she rapidly developed dyspnea, cyanosis and collapse prompting the call of the cardiac arrest team. Despite full anaphylaxis management and resuscitative efforts, including endotracheal intubation, the patient was declared dead 30 minutes after initial collapse. Postmortem examination confirmed a retrocecal diverticular abscess. Serum mast cell tryptase taken immediately postmortem was $>200 \mathrm{ng} / \mathrm{mL}$ (normal range $<14 \mathrm{ng} / \mathrm{mL}$ ).

Case 2: A 27-year-old female, with no significant past medical history, was admitted for induction of labor in her first pregnancy. Due to failure of labor to progress satisfactorily, maternal fatigue and evidence of fetal distress cesarean section was planned. The patient developed a pyrexia of $38^{\circ} \mathrm{C}$ and IV co-amoxiclav $1.2 \mathrm{~g}$ was prescribed preoperatively. Prior to commencement of IV medication the patient confirmed she had no history of drug allergy and had previously tolerated penicillins. During slow IV administration of coamoxiclav the patient complained of a bad taste in her mouth and administration was discontinued. Despite this, symptoms rapidly progressed to include retching, agitation, chest tightness and cyanosis before collapse and cardiac arrest. A consultant anesthetist was in attendance and anaphylaxis management and cardiac resuscitation was commenced immediately. The patient was transferred to theatre and the baby delivered by emergency cesarean section within 15 minutes of initial collapse. The infant showed no signs of life. Exhaustive resuscitative efforts continued and the patient was transferred to the nearest intensive care unit. Despite intensive management she developed left ventricular dysfunction, severe coagulopathy, liver failure and metabolic acidosis. She was declared dead 41 hours after her initial collapse. Serum mast cell tryptase taken
6 hours after the initial reaction was $84.4 \mathrm{ng} / \mathrm{mL}$ (normal range $<14 \mathrm{ng} / \mathrm{mL}$ ).

Case 3: A nine-year-old girl, with a history of atopy including asthma, eczema and food allergy, was admitted to hospital with an infective exacerbation of asthma. She had a history of difficult to manage asthma requiring multiple hospital admissions. During previous admissions she had received IV antibiotics, including IV co-amoxiclav, uneventfully. She had no history of drug allergy but did have peanut allergy. As a result, she and her family had been educated in the symptoms of severe allergic reactions and carried rescue medications including oral antihistamines and epinephrine autoinjectors. On admission, she was tachypnoeic with widespread ronchi. Oxygen saturation was $90 \%$ on room air. Oxygen, nebulised $\beta$ agonists and IV co-amoxiclav (6oo mg tds). Immediately after administration of IV coamoxiclav the child became increasingly distressed. Administration of the dose was completed. Her distress increased rapidly, despite ongoing asthma management, including nebulised epinephrine and salbutamol. Oxygen saturations fell to $58 \%$ on $100 \%$ oxygen. She became deeply cyanosed and collapsed. Full resuscitative efforts were carried out by the cardiac arrest team, but despite this she was declared dead 2 hours and 15 minutes after the administration of coamoxiclav. Serum mast cell tryptase was taken at postmortem examination 2 days after death and was $22.4 \mathrm{ng} / \mathrm{mL}$ (normal range $<14 \mathrm{ng} / \mathrm{mL}$ ).

\section{DISCUSSION}

These cases are presented as confirmed fatal anaphylactic reactions precipitated by the administration of co-amoxiclav; a compound preparation comprising amoxicillin and clavulanic acid. This series highlights a number of important features related to the onset and clinical features of allergy to IV co-amoxiclav, the use of mast cell tryptase as a supportive diagnostic test and the prevalence of drug allergy. The absence of a prior history of drug allergy in all three cases is a notable feature.

The diagnoses in these cases are based on the development of the life-threatening symptoms of anaphylaxis during IV administration of co-amoxiclav. The immediate onset of symptoms is typical of an IgE mediated type I hypersensitivity reaction to a drug [5]. In each case, there was also the supporting evidence of a raised mast cell tryptase. All three patients developed symptoms rapidly during IV administration of the drug. In Cases 1 and 2, where slow administration of coamoxiclav is clearly documented, administration of the dose was stopped as patients complained of an unusual taste in their mouths. This did not prevent the fatal reaction from occurring. In Case 3, the speed of administration is less certain. Onset of symptoms was at completion of the dose and was notable for the presence of a 'sense of impending doom', characteristic of anaphylactic reactions [6]. In each case, acute respiratory failure was the dominant feature. All three 
cases occurred in a hospital setting where expert medical help was immediately available, yet despite exhaustive resuscitative efforts all three patients died. Each patient had a co-factor associated with increased severity of amplification of the reaction; in Case 1 infection, Case 2 pregnancy and Case 3 asthma. These observations emphasize the fact that administration of an intravenous drug in a hospital setting is no guarantee against fatal anaphylactic reactions especially, when potentially exacerbating factors are present.

All three cases presented here occurred in the north of Ireland (Case 2 in Northern Ireland, Case 1 in the Republic of Ireland) over a four year period. In Northern Ireland such reactions are reported via the United Kingdom's Yellow Card System (www.mhra.gov.uk). Their data on amoxicillin, which includes preparations with multiple active ingredients such as co-amoxiclav and Heliclear, indicates that over a 47-year period there were 289 anaphylactic reactions to these drugs. There were only 12 fatalities, 2 of which are presented here. Case 1 occurred in the Republic of Ireland and was therefore not reported via this system. The occurrence of three fatal reactions in a small geographical area, with a relatively small population, is out of proportion to 10 other reported cases in the UK over an extended time frame. The reasons for this are unclear but could include under reporting of fatal allergic reactions, a misclassification of such reactions as asthma deaths or a recent increase in the incidence of such reactions.

Serum mast cell tryptase (sMCT), a marker of mast cell degranulation was elevated in each case, supporting a diagnosis of anaphylaxis [4]. sMCT levels are raised in many, but not all cases, of anaphylaxis related to intravenous medications [7]. Peak SMCT levels occur 1 hour after a reaction and its circulating half life is approximately 2 hours, with levels expected to be normal 24 hours after an allergic reaction. Measurement of sMCT levels is recommended during evaluations of suspected anaphylactic reactions. Although it is notably seldom elevated in food related anaphylaxis [8]. Postmortem sMCT, ideally collected from femoral vessels can be useful in the diagnosis of fatal anaphylaxis [9]. The sMCT is relatively stable in postmortem samples therefore measurement at autopsy may be of value. This information is useful in interpreting the sMCT levels reported in these cases. In Case 1 the tryptase level taken around the time of death was $>200 \mathrm{ng} / \mathrm{mL}$ and is reflective of total serum tryptase levels during the acute reaction. In Case 2 sMCT was $84.4 \mathrm{ng} / \mathrm{mL} 6.5$ hours after the reaction. Based on a two-hour half-life, levels may have been $>500 \mathrm{ng} / \mathrm{mL}$ in the immediate aftermath of the acute reaction. In Case 3 sMCT measurement was only carried out two days postmortem and was only slightly elevated at $22.4 \mathrm{ng} / \mathrm{mL}$. The interpretation of such a value is complex, as recent data suggests that slight elevations in postmortem sMCT can occur especially $>24$ hours after death [9]. Despite this, elevations in sMCT levels, especially $>45 \mathrm{ng} / \mathrm{mL}$, are supportive of a diagnosis of anaphylaxis [9]. However, sMCT is not always elevated in anaphylaxis and fatal reactions to intravenous antibiotics have been associated with only mildly elevated sMCT levels. Measurement of serum mast cell tryptase in the aftermath of anaphylaxis is useful to support the clinical diagnosis.

One of the most striking features of these cases is the absence of a prior history of drug allergy. Indeed, in two cases co-amoxiclav had been administered in preceding weeks without adverse effect. It is routine medical practice to enquire about drug allergies prior to the prescription of antibiotics. It is also recognized that such histories may be unreliable especially as patient reported penicillin allergy often does not reflect the presence or history of allergic sensitization [10]. This has led to the development of algorithms for assessment of drug allergy that can include a combination of clinical history and skin/blood testing for sensitization [2, 10, 11]. Whilst such algorithms may be effective in risk assessing those patients with a history of suspected drug allergy, these cases highlight that a lack of history of a reaction, or indeed recent safe administration of the same medication is no guarantee against subsequent fatal anaphylaxis. Such algorithms are unlikely to have prevented administration of co-amoxiclav or altered outcome in these cases.

\section{CONCLUSION}

These cases illustrate that anyone administering coamoxiclav and other IV antibiotics should be aware of the risk of anaphylaxis, even in the absence of a history of prior reactions. They also demonstrate the catastrophic consequences of anaphylaxis to coamoxiclav, with negative outcomes occurring even in the presence of timely intervention. Further investigation of the prevalence of medication related anaphylaxis fatalities and advances in laboratory methods to aid the clinical diagnosis of such events are required.

$* * * * * * * * *$

\section{Author Contributions}

Niall P Conlon - Conception and design of article, Drafting of article, Final approval of the version to be published

J David M Edgar - Acquisition of data, Aanalysis and interpretation of data, Critical revision of article, Final approval of the version to be published

\section{Guarantor}

The corresponding author is the guarantor of submission.

\section{Conflict of Interest}

Authors declare no conflict of interest.

\section{Copyright}

(C) Niall P Conlon et al. 2013; This article is distributed under the terms of Creative Commons Attribution 3.0 License which permits unrestricted use, distribution and 
reproduction in any means provided the original authors and original publisher are properly credited. (Please see www.ijcasereportsandimages.com /copyright-policy.php for more information).

\section{REFERENCES}

1. Simons FE. Anaphylaxis pathogenesis and treatment. Allergy 2011;66 Suppl 95:31-4.

2. Mirakian R, Ewan PW, Durham SR, et al. BSACI guidelines for the management of drug allergy. Clinical and Experimental Allergy 2009;39(1):43-61.

3. Lee P, Shanson D. Results of a UK survey of fatal anaphylaxis after oral amoxicillin. The Journal of Antimicrobial Chemotherapy 2007;60(5):1172-3.

4. Simons FE. Anaphylaxis. The Journal of Allergy and Clinical Immunology 2010;125(2 Suppl 2):S161-81.

5. Caubet JC, Pichler WJ, Eigenmann PA. Educational case series: Mechanisms of drug allergy. Pediatric Allergy and Immunology 2011;22(6):559-67.

6. Simons FE, Ardusso LR, Bilo MB, et al. World Allergy Organization anaphylaxis guidelines: summary. The Journal of Allergy and Clinical Immunology 2011;127(3):587-93 e1-22.

7. Edston E, van Hage-Hamsten M. beta-Tryptase measurements post-mortem in anaphylactic deaths and in controls. Forensic Science International 1998;93(2-3):135-42.

8. Sampson HA, Mendelson L, Rosen JP. Fatal and near-fatal anaphylactic reactions to food in children and adolescents. The New England Journal of Medicine 1992;327(6):380-4.

9. Mayer DE, Krauskopf A, Hemmer W, Moritz K, Jarisch R, Reiter C. Usefulness of post mortem determination of serum tryptase, histamine and diamine oxidase in the diagnosis of fatal anaphylaxis. Forensic Science International 2011;212(1-3):96-101.

10. McLean-Tooke A, Aldridge C, Stroud C, Spickett GP. Practical management of antibiotic allergy in adults. Journal of Clinical Pathology 2011;64(3):192-9.

11. Rosario NA, Grumach AS. Allergy to beta-lactams in pediatrics: a practical approach. Jornal de Pediatria 2006;82(5 Suppl):S181-8.
Access full text article on other devices

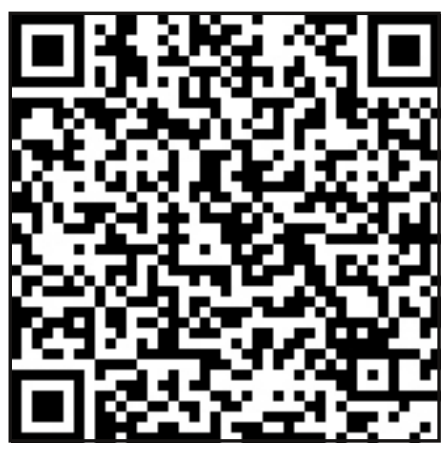

Access PDF of article on other devices

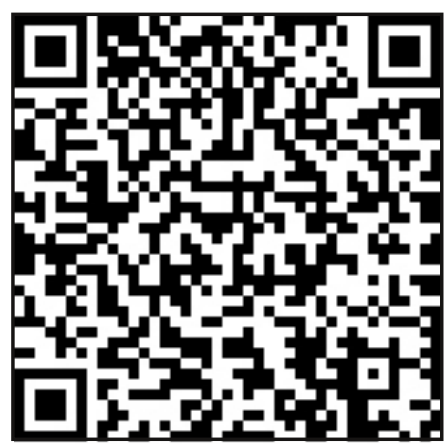

